\title{
Acerca de los efectos de la localización de la nueva vivienda social sobre las luchas por el acceso a la ciudad de los sectores populares
}

Mercedes Najman. Universidad de Buenos Aires, Buenos Aires, Argentina.

RESUMEN | Recientemente, en la Ciudad de Buenos Aires se implementaron diversas políticas habitacionales "novedosas" que, aunque continuaron emplazándose mayormente en zonas desfavorecidas, incorporaron áreas más ventajosas como lugares posibles para la vivienda social. Este artículo analiza los efectos de la localización desigual de la nueva vivienda social sobre las inscripciones territoriales de sus destinatarios. Mediante un análisis de sus trayectorias residenciales, reflexiona sobre la capacidad de estas políticas para transformar las posiciones que estos hogares ocuparon históricamente en la estructura urbana. Los resultados dan cuenta de que, indistintamente de su localización, la vivienda social tiende a reproducir las inscripciones territoriales previas de sus destinatarios, sin imprimir efectos positivos sobre sus trayectorias residenciales ni trastocar el lugar de los sectores populares en la estructura urbana. Al habilitar accesos diferenciales a la ciudad por medio de distintas localizaciones, las nuevas políticas de vivienda social terminan reproduciendo y profundizando las desigualdades urbanas vigentes.

PALABRAS CLAVE | localización, movilidad, política habitacional.

ABstract | Recently, in the City of Buenos Aires a series of "novel" housing policies were developed, which incorporate more advantageous areas as possible places for social housing, although they continued to be located mostly in disadvantaged areas. This article analyzes the effects of the unequal location of the new social housing on the territorial inscriptions of its inhabitants. Using an analysis of their residential trajectories, we reflect on the capacity of these policies to transform the positions that these households historically occupied in the urban structure. The results show that regardless of its location, social housing tends to reproduce the previous territorial inscriptions of its inhabitants, without printing positive effects on their residential trajectories or disrupting the place of the popular sectors in the urban structure. By enabling differential accesses to the city through different locations, the new social housing policies worsen urban inequalities.

KEYWORDS | location, mobility, housing policy. 


\section{Introducción}

Este artículo reflexiona acerca de ciertos efectos territoriales que la localización de la vivienda social genera sobre sus habitantes, interrogando la capacidad de estas políticas para transformar las condiciones de acceso a la ciudad de sus destinatarios. Si bien, en términos cuantitativos, la tradición de producción de vivienda social en el país y la ciudad estuvo destinada a sectores trabajadores formales, aquí nos centramos particularmente en aquellas intervenciones que se orientaron a dar una solución habitacional a poblaciones que, hasta el momento, habían resuelto sus necesidades habitacionales mediante mecanismos informales. Particularmente, se pone la mirada en torno a las recientes políticas de vivienda social desarrolladas en la Ciudad Autónoma de Buenos Aires (CABA), bajo el propósito de iluminar los quiebres y continuidades que presentan en relación con los efectos territoriales de las denominadas "políticas tradicionales de vivienda social".

En los ańos sesenta, se levantaron en la ciudad las primeras viviendas sociales destinadas a grupos poblacionales que residían en situaciones informales. Fueron construidas bajo discursos fuertemente moralizantes y en tipologías arquitectónicas de grandes conjuntos urbanos en áreas relegadas de la ciudad (Girola \& Thomasz, 2013; Massidda, 2011; Snitcofsky, 2013). Numerosos estudios dieron cuenta de las críticas que despertaron estas primeras intervenciones, principalmente a partir de los efectos negativos de su localización, la intensificación de la segregación socioresidencial de sus habitantes y el desarrollo de nuevos estigmas territoriales (Ballent, 1998; Dunowicz \& Boselli, 2009; Girola, 2008; Girola \& Thomasz, 2013). Como consecuencia de estas críticas y en el marco de la reforma del Estado, hacia finales de los años setenta se produjo una merma en la producción estatal de vivienda (Ballent, 2018).

Recién a inicios del 2000 aparece un renovado impulso en la construcción de vivienda social para sectores populares, aunque bajo nuevos paradigmas (Rodríguez, 2012; Rodulfo, 2008; Rodulfo \& Boselli, 2015; Varela \& Fernández Wagner, 2012). En este contexto, la implementación de una política de vivienda, entendida como un engranaje indispensable para la recuperación del desarrollo económico y como canal hacia la integración social mediante la conjunción de los ejes techo y trabajo, fue planteada como un objetivo prioritario (Rodríguez, 2012). Las nuevas soluciones habitacionales destinadas a quienes se inscribían informalmente en la ciudad asumieron varias de las críticas realizadas a las políticas tradicionales y, si bien buscaron saldar algunos de estos elementos -resaltamos la aparición de nuevas tipologías arquitectónicas y, principalmente, de nuevas localizaciones en la ciudad-, dejaron entrever ciertas continuidades sobre el modo de producir vivienda social para los sectores vulnerables.

Los efectos que estas "nuevas" formas de producir vivienda social despiertan sobre las condiciones de acceso a la ciudad de sus habitantes permanecen como interrogantes aún no resueltos. ¿En qué tipo de localizaciones se desarrollaron las nuevas viviendas sociales? Estas "nuevas" posiciones, ¿̨habilitaron el acceso a mejores externalidades urbanas? ¿De qué manera la localización de la vivienda social afectó 
el desenlace de las disputas territoriales que estos hogares protagonizaron a lo largo de sus biografías?

Este artículo presenta los resultados de un estudio comparado de corte cuantitativo y longitudinal, que analiza el papel desempeñado por la localización de la nueva vivienda social sobre las trayectorias residenciales de sus destinatarios a partir de dos casos de estudio, desarrollados recientemente en dos zonas contrastantes de la ciudad. La investigación se enfoca en términos metodológicos, pero también en tanto encuadre teórico, sobre los cursos de vida de los hogares destinatarios de la vivienda social, anclando en su dimensión territorial. A partir del análisis comparativo de viviendas sociales con distinta localización, se interroga el potencial que estas intervenciones tienen para modificar las posiciones que sus destinatarios ocuparon históricamente en la estructura urbana.

\section{Acerca de las posiciones urbanas, las luchas por el acceso a la ciudad y el rol de la vivienda social}

El espacio urbano es resultado de prácticas e interacciones, de relaciones de poder, de conflictos y estrategias de diferentes actores, y si bien se nos presenta como una realidad externa y objetiva, se trata de un producto eminentemente social (Massey, 2005). Pero, ¿cómo se configuran las estructuras urbanas y por qué operan como instancias (re)productoras de la desigualdad social?

Retomando a Oszlak (1991), entendemos la estructura urbana como el resultado de un proceso social que define la distribución geográfica -desigual- de los bienes, servicios y grupos sociales en el espacio. Las luchas permanentes por el derecho a la ciudad que atraviesan los procesos de configuración de la estructura urbana, no solo dan cuenta de una disputa en torno a la disposición del espacio urbano, sino también respecto a su uso (Oszlak, 1991). Esto significa que, si bien la ciudad constituye un sistema de recursos utilizables, al estar estos bienes y servicios desigualmente distribuidos, se producen situaciones de desigualdad en torno a las oportunidades asociadas a la ciudad.

Tal como argumenta Harvey (1977), a cada posición territorial o localización se le asocian distintos niveles de proximidad y acceso a los recursos urbanos, dando lugar a lo que Flores (2008) denomina "geografías de oportunidades" diferenciales, las cuales influyen directamente sobre las condiciones de vida de sus habitantes al intervenir sobre sus estructuras de oportunidades (Kaztman, 1999, 2000). Como consecuencia, las posiciones que los hogares ocupan en la estructura urbana $-y$ también en la estructura social- condicionan su capacidad de apropiarse del espacio y gozar de sus externalidades positivas (Bourdieu, 1999).

Desde ya, no todos los hogares tienen las mismas posibilidades de acceder a las áreas más favorables de la ciudad. Los márgenes de acción de los hogares en materia de hábitat se ven limitados o condicionados por factores estructurales, tales como la dinámica del mercado de suelo y vivienda, pero también por otros elementos, como el mercado de trabajo y las políticas de vivienda vigentes (Dieleman, 2001; Pooley, 1997; Slater, 2013). La segmentación del espacio social no solo es atribuible a la distribución geográficamente desigual de las externalidades urbanas, sino también 
a la condición mercantilizada del espacio urbano. Tal como sostienen Del Río et al. (2014), el alza de precios de tierra y vivienda en las zonas con mejor acceso a bienes y servicios genera una barrera económica para el acceso de las familias de sectores populares a las posiciones urbanas más codiciadas.

Sin embargo, sería erróneo concebir las posiciones residenciales como elementos estáticos (Clark, 1982; Dureau, 2003; Jirón \& Imilan, 2018). Los procesos de movilidad residencial -prácticas espaciales que introducen cambios en el lugar de residencia de un hogar (Di Virgilio, 2007) ${ }^{1}$ - dan cuenta de las disputas que, a lo largo de sus vidas, los hogares lideran en torno al derecho al acceso y uso de las externalidades positivas que brinda la ciudad. Así, las trayectorias residenciales (en tanto encadenamiento sucesivo de las prácticas de movilidad residencial) vuelven legibles los éxitos o fracasos de estas disputas biográficas por el espacio urbano (Bourdieu, 1999). A lo largo de este artículo nos proponemos analizar en esta clave las trayectorias residenciales de quienes llegan a las nuevas viviendas sociales, con la intención de identificar la incidencia de estas intervenciones sobre el desenlace de sus luchas por el espacio urbano.

Este interrogante se desprende del rol que asume el Estado en los procesos de configuración de la estructura urbana, ya que a través de sus acciones -entre ellas, la producción de vivienda social- tiene la capacidad de transformar las estructuras urbanas excluyentes y, en consecuencia, modificar las condiciones de vida de la población (Del Río, 2010; Oszlak, 1991; Rodríguez \& Di Virgilio, 2011; Rodríguez \& Sugranyes, 2011). Esta "capacidad transformadora" de las políticas de vivienda social reposa en gran medida sobre su localización, constituyendo una dimensión crítica para la definición de la calidad de tales políticas (Del Río, 2010; Hidalgo, 2007; Hidalgo et al., 2007). Dependiendo del lugar que las viviendas sociales ocupan en la estructura urbana, promueven o impiden la capacidad de sus destinatarios de gozar de las oportunidades y los valores de uso complejo que ofrece la ciudad. Aun localizándose en áreas deficitarias, tal como señala Baumont (2007), las políticas de vivienda también tienen la capacidad de actuar sobre las características -objetivas y subjetivas- de las áreas donde se implementan, trastocando de esta forma la posición relativa de un lugar mediante una redistribución de las externalidades urbanas.

A pesar de la potencialidad transformadora que reposa sobre la localización de la vivienda social, las experiencias latinoamericanas - entre ellas, las políticas tradicionales desarrolladas en la Ciudad de Buenos Aires- dan cuenta de que la ubicación de estas intervenciones contribuyó a intensificar y reproducir la desigualdad de los patrones espaciales preexistentes (Dunowicz \& Boselli, 2009; Rodríguez \& Sugranyes, 2005; Ziccardi \& Mier y Terán, 2015). Cabe destacar que en la CABA se cristaliza un patrón espacial desigual que divide la ciudad en dos grandes ejes: norte y sur. La desigualdad entre estos dos sectores es resultado de procesos de larga data, y se evidencia actualmente en los valores del suelo y de los inmuebles, en los patrones de distribución residencial de la población y en la geografía de los equipamientos e infraestructuras urbanas (Di Virgilio et al., 2019; Fachelli et al., 2015; Gorelik \& Silvestri, 1991).

1 En este trabajo nos centramos en la dimensión espacial de estas prácticas, considerando los cambios que ellas producen (o no) sobre las posiciones urbanas. 
Ahora bien, la priorización histórica del sector sur de la ciudad como localización de la vivienda social (Ferme et al., 2017; Najman, 2020; Rodulfo \& Boselli, 2015) implicó no solo la reproducción de las características desiguales de la estructura urbana, sino también la intensificación de las mismas mediante la concentración residencial de población de bajos recursos (y grandes necesidades) en un territorio con escaso acceso a las externalidades urbanas necesarias para satisfacer sus demandas. En este sentido, tal como afirman Carman et al. (2013), podemos considerar que las políticas habitacionales para población vulnerable han tendido a producir y reproducir procesos de segregación socio-residencial a través de lo que los autores denominan como un proceso de segregación acallada, al confinar espacialmente a los sectores más débiles, de manera implícita, bajo una medida asistencial o una política de inclusión social.

Frente a las numerosas críticas esgrimidas internacionalmente respecto de los efectos negativos que una localización deficitaria de la vivienda social puede tener sobre la concentración territorial de la pobreza, en las últimas décadas, tanto en Estados Unidos como en diversos países de Europa se comenzaron a implementar programas habitacionales alternativos que buscaban solucionar tales efectos mediante políticas de "mixtura social residencial". En años recientes, estas perspectivas han comenzado a encontrar eco en algunos programas habitacionales en nuestra región (RuizTagle \& Romano, 2019; Vergara Erices, 2019), y de manera incipiente en la CABA, como lo demuestra uno de nuestros casos de estudio. En este sentido, las políticas de vivienda social más recientes en las que aquí nos enfocamos, si bien tendieron a ocupar los mismos lugares en la ciudad que aquellas desarrolladas previamente, incorporaron en cierta medida localizaciones disruptivas para su construcción, algunas de las cuales se ubican en el eje norte de la ciudad (Figura 1).

\section{FIGURA I | Distribución de la vivienda social destinada a sectores populares por periodo histórico, CABA}

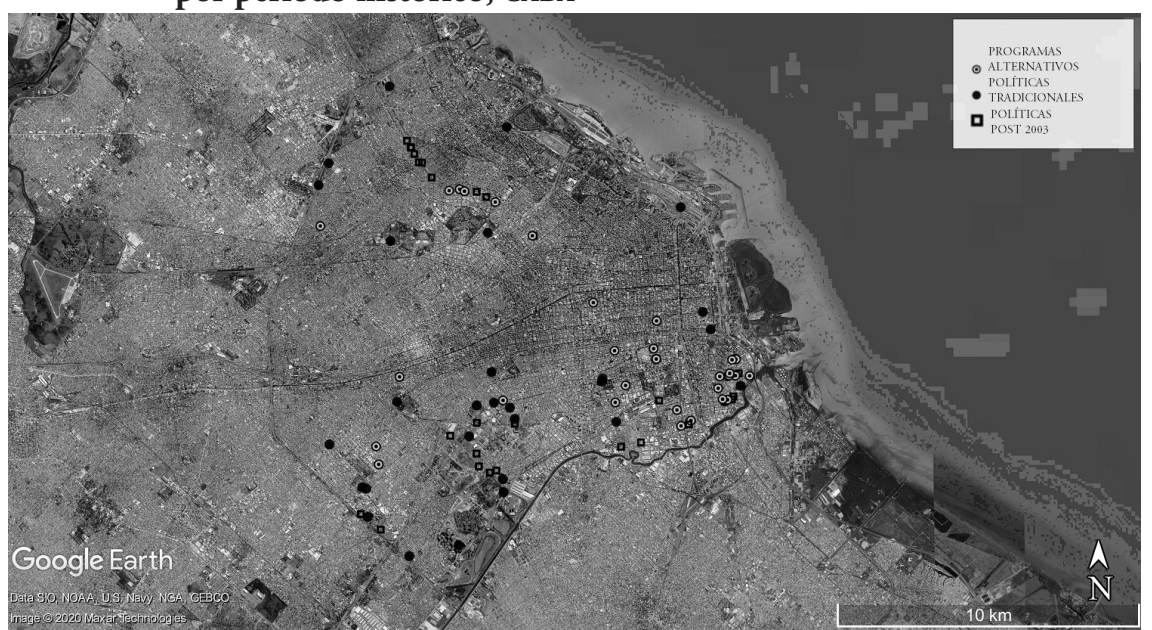

FUENTE: ELABORACIÓN PROPIA SOBRE MAPA DE GOOGLE EARTH 
El desarrollo reciente de políticas de vivienda social en dos zonas contrastantes de la ciudad (norte y sur) habilita nuevos interrogantes en torno al rol que desempeña la localización de estas viviendas sobre la capacidad de las políticas de transformar las condiciones de acceso a la ciudad de sus habitantes. En consecuencia, en este trabajo nos preguntamos en qué medida estas dos localizaciones habilitaron una mejora en las posiciones que estos hogares ocupaban previamente en la ciudad. En otras palabras, interesa comprender el impacto de la nueva vivienda sobre las luchas por el acceso a la ciudad que estos hogares desarrollaron a lo largo de su vida. ¿Desde dónde llegaron a la vivienda social? ¿Cuáles habían sido los desenlaces anteriores de sus disputas territoriales? ¿Qué papel desempeñó sobre estos recorridos el ingreso a la vivienda social? ¿Qué lugar ocupa la vivienda social no solo en la estructura urbana, sino también en las geografías que dibujan las trayectorias residenciales de sus habitantes?

\section{Un estudio comparado desde un abordaje cuantitativo y biográfico}

Esta investigación se inscribe en el campo interdisciplinario de los estudios urbanos y del hábitat y se enfoca en términos metodológicos, pero también en tanto encuadre teórico, sobre las trayectorias residenciales de los hogares destinatarios de la vivienda social, anclándose en su dimensión biográfica y territorial (Di Virgilio \& Najman, 2019). En este sentido, se exploran los efectos diferenciales de la localización de la vivienda social, considerando el acceso a oportunidades urbanas que las distintas posiciones habilitan, no solo desde una perspectiva sincrónica, sino principalmente tomando en cuenta los efectos de la localización de la vivienda social sobre las trayectorias residenciales de sus habitantes. Persiguiendo este objetivo se desarrolló una investigación comparativa en torno a dos casos de estudio -los conjuntos habitacionales Padre Mugica y de la ex AU3- que, al localizarse en el eje norte y sur de la ciudad (Figura 2), permiten considerar el rol que ejerce el lugar de la vivienda social sobre la capacidad de garantizar el derecho de sus habitantes a la ciudad y al espacio urbano.

El barrio Padre Mugica se ubica en el denominado eje sur de la ciudad, más precisamente en Villa Lugano, Comuna 8. Se trata de una zona que concentra altos niveles de precariedad habitacional y vulnerabilidad social, superando ampliamente los valores promedio de la ciudad (Consejo Económico y Social de la CABA, 2013). Este nuevo barrio no solo se ubica en el eje sur de la ciudad, sino que lo hace en una zona caracterizada por la presencia de proyectos anteriores de vivienda social y de hábitats populares que permiten pensarla como un "enclave de pobreza" (Carman et al., 2013). Se trata de un complejo urbano de 780 viviendas sociales, construido inicialmente a través del Programa Sueños Compartidos en el marco de los Planes Federales de Construcción de Vivienda. Si bien su localización en el eje sur de la ciudad reproducía los patrones espaciales de las tradicionales viviendas sociales (Figura 1), en el diseńo original de esta política se evidenciaba cierto impulso de dotación de esta área de la ciudad históricamente deficitaria: se incorporaba la construcción de barrios con escuelas, centros de salud, entre otros bienes, dando cuenta con ello de una impronta "equitativa" o "igualadora" de una estructura urbana 
desigual. No obstante, estos lineamientos iniciales fueron desdibujándose en el proceso de implementación del proyecto, y finalmente la construcción se llevó a cabo sin la dotación de equipamientos y servicios previstos inicialmente, dejando como saldo la intensificación de la característica deficitaria de la zona en la cual se emplazó (Najman, 2017). Asimismo, aunque la localización del barrio en el proyecto inicial respondía a una impronta de radicación de la población que residía previamente en esta zona bajo modalidades informales, en el proceso de adjudicación se produjo un nuevo desfasaje y los habitantes del nuevo barrio debieron atravesar procesos de relocalización desde distintas villas y asentamientos de la ciudad, que involucraron el desarraigo y, en muchos casos, el deterioro de su posición en la estructura urbana, como lo veremos a continuación.

FIGURA 2 | Localización de los casos de estudio en la caba

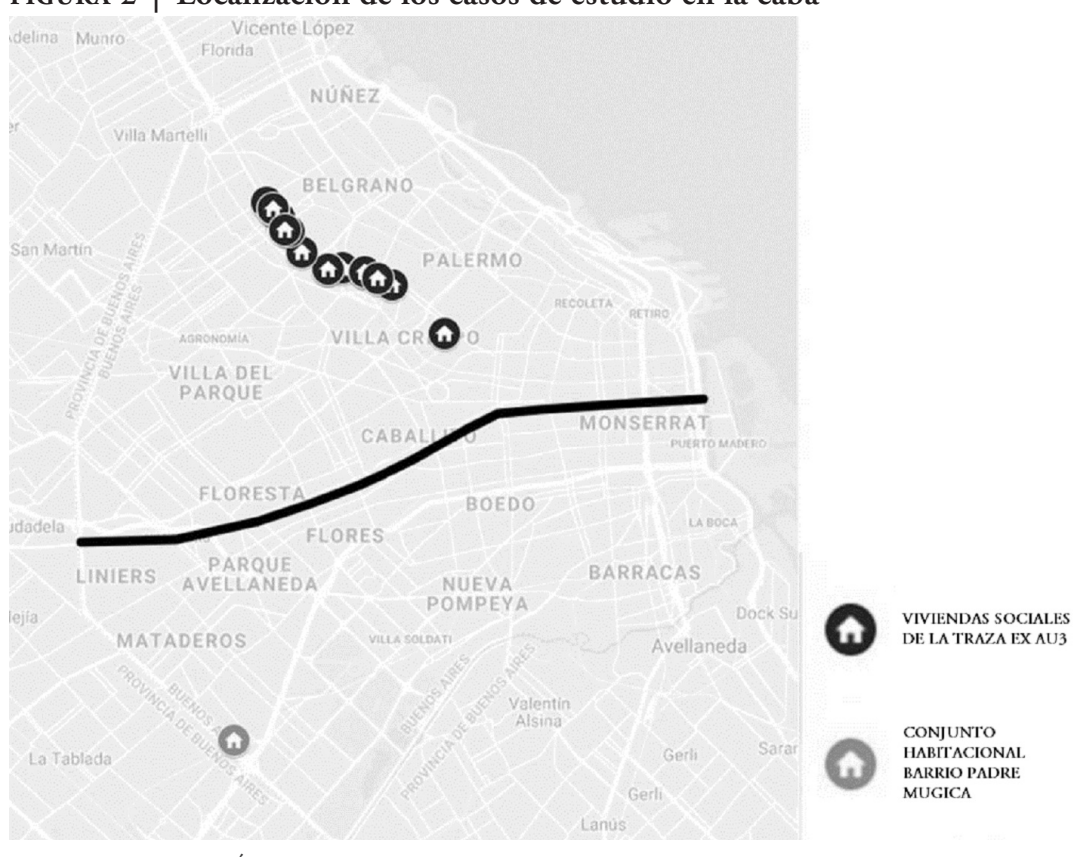

FUENTE: ELABORACIÓN PROPIA

En contraste, las viviendas sociales de la ex AU3 (Autopista número 3) se ubican en el eje norte de la ciudad de forma dispersa, atravesando los barrios de Villa Urquiza, Villa Ortúzar y Chacarita, espacios no privilegiados previamente para la producción de vivienda social. Esta zona de la ciudad estuvo históricamente poblada por sectores medios y actualmente atraviesa un proceso de fuerte renovación y revalorización (Cavalieri et al., 2010). Asimismo, a diferencia del barrio Padre Mugica, el entorno que rodea a estas viviendas no se caracteriza por la presencia de otras soluciones habitacionales ni asentamientos informales, sino que se trata de un entorno urbano principalmente formal. De hecho, este conjunto de viviendas sociales fue construido con el propósito de "resolver" las modalidades informales de ocupación (excepcionales en la zona) en las que residían sus destinatarios. 
Las viviendas sociales construidas en la traza de la ex AU3 se inscriben en un proyecto urbano que busca dar solución a una disputa territorial iniciada durante la última dictadura militar. El Plan de Autopistas Urbanas, entre las cuales se encontraba la Autopista número 3 (AU3) que atravesaría la ciudad de norte a sur, impulsó la expropiación y demolición de las viviendas que se encontraban en el trayecto de la traza proyectada, dividida en distintos "sectores". Pese a que la AU3 no fue finalmente concretada, se alcanzó a expropiar aproximadamente 800 inmuebles, aunque este proceso adoptó características particulares en cada uno de los "sectores" de la traza (Pérez Ripossio, 2013), dando lugar, en los sectores 4 y 5, a procesos de ocupación de dichos inmuebles inmediatamente posteriores a la expropiación (Najman, 2020; Zapata \& Belluscio, 2018). El abandono del proyecto de construcción de la autopista dio pie al surgimiento de un conflicto urbano que tendría una larga duración y sobre el cual el Estado intervino de manera heterogénea a lo largo del tiempo. Las viviendas sociales de la ex AU3 se desarrollaron a partir de la Ley $324 / 1999,^{2}$ la cual contemplaba la creación del Programa de Recuperación de la Traza de la ex AU3. Este proponía solucionar la problemática habitacional de aquellas familias que habitaban las viviendas ocupadas y censadas en la traza, a la vez que buscaba reconstruir el tejido urbano, dando cuenta de un impulso estatal sobre los procesos de renovación y valorización de este sector de la ciudad (Najman, 2020).

Pese a que el origen del programa de la ex AU 3 antecede en casi una década al otro caso de estudio, el del barrio Padre Mugica, la construcción de estas viviendas cobró un impulso real gracias al desarrollo del Plan Federal de Vivienda, que habilitó nuevas modalidades de financiamiento local. De esta forma, casi simultáneamente a la construcción del barrio Padre Mugica, se desarrollan cinco soluciones habitacionales inscriptas en la Ley 324/1999, y posteriormente cinco conjuntos "llave en mano" y tres edificios reciclados a partir de las leyes 3396/20093 y 4089/2011, ${ }^{4}$ estas últimas amparadas en un discurso orientado a la "mixtura social" de la zona.

Sin embargo, a pesar de ser dos soluciones habitacionales contemporáneas, sus localizaciones fueron claramente contrastantes. La localización de las viviendas sociales de AU3 no solo fue disruptiva, al ubicarse en el eje norte de la ciudad, sino que, además, habilitó la radicación de aquellos hogares que residían previamente allí de manera informal. Cabe aclarar que estos elementos novedosos no dan cuenta de una transformación sobre la definición de las políticas habitacionales locales, sino que la producción de vivienda social orientada a la radicación de los hogares

2 La Ley 324/1999 es resultado de un fuerte proceso de organización de los hogares ocupantes de la traza frente a intentos de desalojo. La resistencia consolidó un entramado actoral con capacidad de presión, que posibilitó la sanción de una ley que incorporaba una impronta de radicación de los hogares ocupantes.

3 A partir del año 2007 se reanudaron los intentos de desalojo de los hogares ocupantes, despertando nuevas resistencias. En respuesta, el Gobierno de la Ciudad de Buenos Aires impulsó la sanción de una nueva ley que fue foco de un complejo debate legislativo con alta participación de los afectados. Finalmente, la Ley 3396/2009 volvió a ofrecer distintas alternativas para dar solución a la problemática habitacional, principalmente en el sector 5 .

4 La Ley 4089/2011 es modificatoria de la Ley 3396/2009 y la 324/1999, y persigue el objetivo de utilizar los fondos "sobrantes" de la renovación urbana del sector 5, para concretar las soluciones habitacionales previstas en la Ley 324 del sector 4. 
ocupantes en la traza surgió como respuesta a un fuerte proceso organizativo de resistencia al desplazamiento (Rodríguez, 2005). En este sentido, mientras que el barrio Padre Mugica fue poblado mediante procesos de relocalización desde otras zonas de la ciudad hacia un área desfavorecida, los habitantes de las viviendas sociales de la ex $\mathrm{AU} 3$ atravesaron un proceso de radicación en una zona disputada y con mayores externalidades urbanas.

Los procesos de poblamiento de ambos casos de estudio dan cuenta de la relevancia de considerar los efectos del lugar residencial no solo desde una perspectiva sincrónica, sino principalmente diacrónica y longitudinal, identificando el rol que asume el lugar de la vivienda social en el marco de los recorridos residenciales anteriores en tanto luchas por el acceso a la ciudad. Con tal objetivo, esta investigación comparativa se inscribe en un encuadre cuantitativo y biográfico desarrollado a partir de datos primarios obtenidos con encuestas longitudinales -Encuesta de Movilidad Espacial (EME) ${ }^{5}$ - aplicadas en un conjunto de hogares representativos de los dos casos de estudio. Del total de la muestra se trabajó con 160 casos y se reconstruyeron las trayectorias residenciales mediante los calendarios biográficos completados por "ego" (representante de cada hogar).

Mediante un análisis de las secuencias (Gauthier et al., 2010; Ritschard \& Studer, 2018) que conforman los movimientos residenciales de los encuestados a lo largo de su vida, se reconstruyeron las trayectorias residenciales de localización para un representante de cada hogar desde su nacimiento hasta el ingreso a la vivienda social (considerado este último como tiempo "0" (cero)). Los recorridos fueron truncados al momento de ingresar a la vivienda social, por lo que las trayectorias tienen duraciones heterogéneas en función de la edad en que ingresa cada "ego" a la vivienda social.

Las trayectorias señaladas hacen visibles los recorridos residenciales a partir de cuatro categorías de jerarquización del espacio al que accedieron las familias, definidas en relación con el centro de la ciudad y el valor del suelo: i) Otros países o provincias; ii) Gran Buenos Aires (GBA); iii) Barrios de CABA desvalorizados; iv) Barrios de CABA más valorizados. ${ }^{6}$ Junto a las posiciones urbanas de estas residencias, nos preguntamos acerca del modo en que los hogares se inscribieron en el territorio. De esta forma, consideramos la localización residencial articulando tanto la ubicación -relativa- como las características del hábitat (Marcos et al., 2015) o del mercado de tierra y vivienda por el cual los hogares accedieron a tales posiciones (mercado formal, informal, o bien soluciones provistas por el Estado).

A continuación se presentan los resultados de la investigación en dos secciones. En una primera instancia se analizan las trayectorias previas a la vivienda social, evidenciando los distintos derroteros de las luchas por la apropiación del espacio urbano que estos hogares habían protagonizado anteriormente. Luego, la segunda

5 La Encuesta de Movilidad Espacial (EME) es realizada en Buenos Aires por el Área de Estudios Urbanos del Instituto de Investigaciones Gino Germani (IIGG).

6 La división de la CABA en dos fracciones se corresponde con la división ya mencionada norte-sur y mediante el valor de los inmuebles promedio en cada barrio (Reporte de mercado inmobiliario, Properati, 2019). 
sección se enfoca en la llegada a la vivienda social, presentando los efectos que esta nueva localización desencadenó sobre aquellos recorridos.

\section{Las luchas por la apropiación de la ciudad que traducen las trayectorias residenciales}

El análisis de las trayectorias residenciales que los destinatarios desarrollaron desde su nacimiento hasta el momento previo a ingresar a la vivienda social, visibiliza los diversos desenlaces de las luchas que estos hogares protagonizaron para gozar de las oportunidades y los valores de uso complejo que ofrece la ciudad. Las características de estas trayectorias - principalmente las posiciones que estos hogares ocuparon previamente en la estructura urbana y la orientación de estos recorridos en clave de lucha por el acceso a la ciudad- resultan indispensables para comprender el rol que asume la localización de la vivienda social en las disputas territoriales que los destinatarios encarnaron a lo largo de sus vidas.

El análisis de estas trayectorias no solo evidencia la heterogeneidad al interior de un grupo usualmente indiferenciado bajo el rótulo de "destinatarios de la vivienda social", sino que, además, presenta patrones diferenciados entre quienes terminan siendo habitantes de las soluciones habitacionales localizadas en el eje norte de la ciudad, o aquellos ubicados en el eje sur.

\section{FIGURA 3 | Ubicación de residencias anteriores, en porcentajes según barrio de vivienda social de destino}

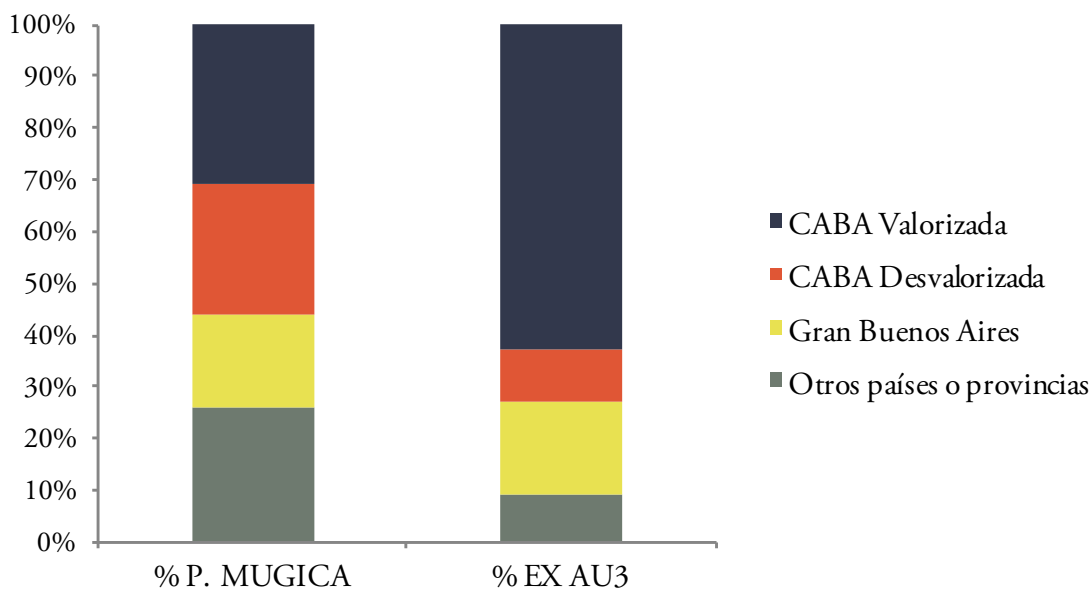

FUENTE: ELABORACIÓN PROPIA CON BASE EN LA ENCUESTA DE MOVILIDAD ESPACIAL (20I6-20I9)

En primer lugar, la ubicación del conjunto de residencias anteriores de estos destinatarios demuestra un uso residencial del espacio muy diverso en las trayectorias de los habitantes de los dos casos de estudio (Figura 3). La zona más valorizada de la ciudad -y con mayor acceso a externalidades positivas- estuvo muy presente en las trayectorias de quienes llegaron a $\mathrm{AU}_{3}$, y si bien el mayor porcentaje de etapas 
residenciales se da entre los futuros habitantes de Padre Mugica, en términos relativos es considerablemente menor que el de los primeros. A su vez, a diferencia de los habitantes de $\mathrm{AU} 3$, quienes llegan a Mugica presentan trayectorias con mayor presencia en los barrios desvalorizados de la CABA y en otros países o provincias, dando cuenta de la relevancia de los procesos migratorios.

Por otro lado, mientras quienes llegaron a la vivienda social en AU3 habían priorizado previamente esa zona como un espacio de vida (más del $46 \%$ de los movimientos residenciales de esta población se emplazaron en la zona de la traza), por el contrario, solo el $4 \%$ de los movimientos residenciales de quienes llegaron a la vivienda social ubicada en el sur se habían localizado en el barrio Villa Lugano. Esta abrumadora diferencia da cuenta de los espacios privilegiados por estos hogares (y posibles) para residir, a la vez que evidencia que no siempre coinciden con los lugares considerados por el Estado para desarrollar la vivienda social.

Las posiciones residenciales anteriores de estos dos casos de estudio no solo muestran un acceso desigual a la ciudad en términos de alcance de mejores posiciones urbanas, sino que también presentan patrones diferenciales en torno a los mecanismos de acceso a estos territorios (Figura 4).

FIGURA 4 | Tipo de inserción territorial en cada ubicación, en porcentajes según barrio de vivienda social de destino

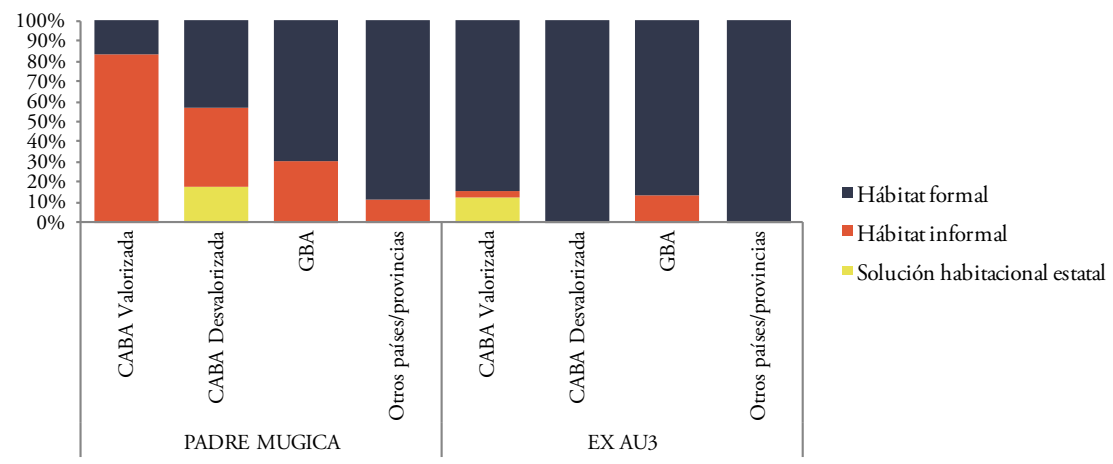

FUENTE: ELABORACIÓN PROPIA CON BASE EN LA ENCUESTA DE MOVILIDAD ESPACIAL (20I6-20I9)

Los recorridos residenciales de quienes llegaron a Mugica presentan una relación casi inversa entre posición urbana y modo de inscripción territorial: la informalidad parece haber funcionado como el canal de acceso a las mejores localizaciones, principalmente a los barrios más valorizados de la CABA. En este sentido, los mercados informales funcionaron para estos hogares como un mecanismo para superar la barrera económica que impide el acceso de los sectores populares a las posiciones urbanas más codiciadas (Del Río et al., 2014). Por el contrario, los habitantes de AU3 no muestran trayectorias en que los hábitats informales se presenten como estrategias relevantes de acceso al suelo y a la vivienda. Como mencionábamos previamente, la informalidad territorial estuvo presente para estos hogares mediante mecanismos más atomizados e invisibilizados, como la ocupación de inmuebles. 
Ahora bien, para comprender las luchas residenciales que los hogares protagonizaron por la apropiación del espacio urbano a la luz de estos recorridos, debemos incorporar la dimensión temporal y secuencial de estas trayectorias. ¿¿Desde dónde y hacia dónde se han movido estos hogares? Los movimientos residenciales, ¿̨an cuenta de mejoras o retrocesos en relación con el acceso a mejores externalidades urbanas?

Mediante el análisis de secuencias de las trayectorias de localizaciones desde el momento del nacimiento de sus protagonistas hasta el acceso a la vivienda social, podemos dar cuenta no solo de las presencias residenciales en las distintas localizaciones de la estructura urbana, sino del orden en que se dan estos movimientos, así como del tiempo de permanencia en cada una de estas posiciones, pudiendo identificar los derroteros de las luchas por el acceso a la ciudad.

FIGURA 5 | Secuencias de trayectorias residenciales individuales por año de vida de los habitantes de AU3

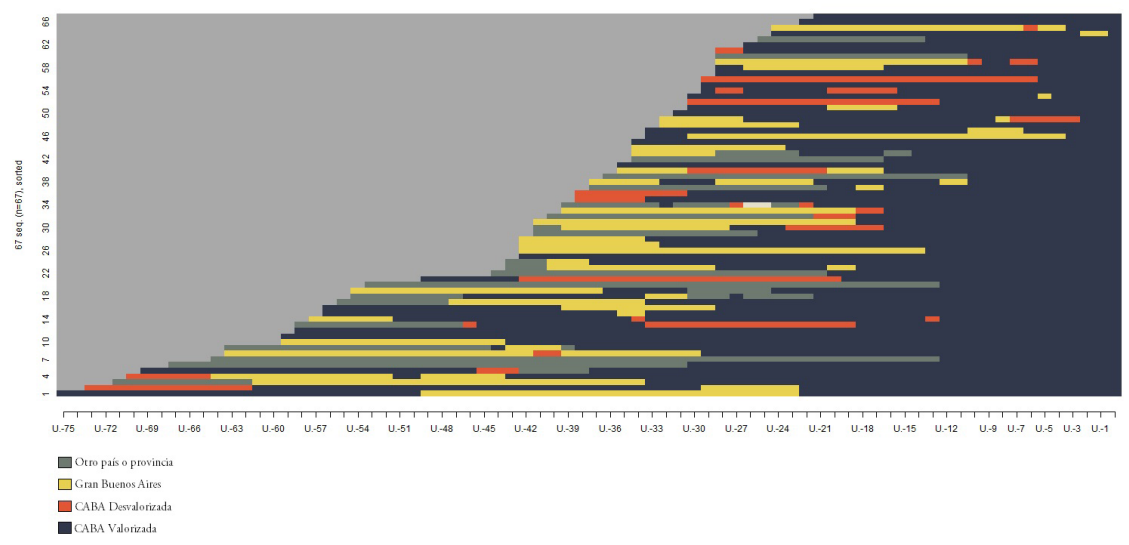

FUENTE: ELABORACIÓN PROPIA CON BASE EN LA ENCUESTA DE MOVILIDAD ESPACIAL (20I6-20I9)

FIGURA 6 | Tipos de trayectorias de localización residencial de los habitantes de $\mathrm{AU} 3$, en porcentajes

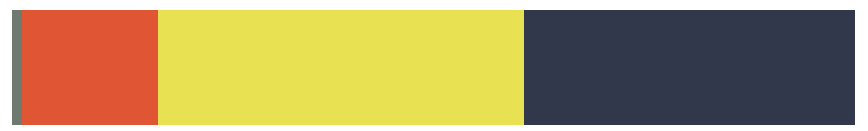

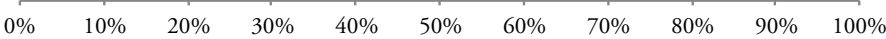

- Descendentes

- Lineales

Oscilantes

Ascendentes

FUENTE: ELABORACIÓN PROPIA CON BASE EN LA ENCUESTA DE MOVILIDAD ESPACIAL (20I6-20I9) 
Las trayectorias que culminan en la vivienda social de la ex AU 3 se desarrollaron principalmente sobre los barrios más valorizados de la CABA; allí permanecen los hogares durante gran parte de su existencia e, incluso, desde muy temprano (Figura 5). Pero antes que trayectorias lineales (donde a lo largo de toda la vida se conserva una misma posición residencial, en este caso favorable), quienes llegan a estas viviendas sociales muestran recorridos principalmente oscilantes y ascendentes (Figura 6). Sus trayectorias demuestran que han logrado acceder a las zonas con mayores externalidades urbanas, provenientes de otras localidades de GBA o desde otros países o provincias, y mejorando con ello sus posiciones urbanas. Pero el elevado porcentaje de trayectorias oscilantes da cuenta de que estos caminos ascendentes han sido complejos e inestables, y se caracterizaron por experimentar avances y retrocesos en esta "escalera residencial".

Así, la priorización de las zonas más valorizadas de la ciudad como espacio de vida por estas trayectorias, parece involucrar necesariamente escenarios de lucha y conquista por mejores posiciones urbanas, pero también sugiere la dificultad de estos hogares para sostener estas conquistas, las cuales son evidentemente provisorias.

En este marco, el acceso a la vivienda social emplazada en una localización que sus destinatarios habían priorizado previamente para residir, podría funcionar como un factor de estabilidad para estos hogares, cuyas trayectorias oscilantes demostraron una búsqueda continua por permanecer en esta zona.

\section{FIGURA 7 | Secuencias de trayectorias residenciales individuales por año de vida de los habitantes de Padre Mugica}

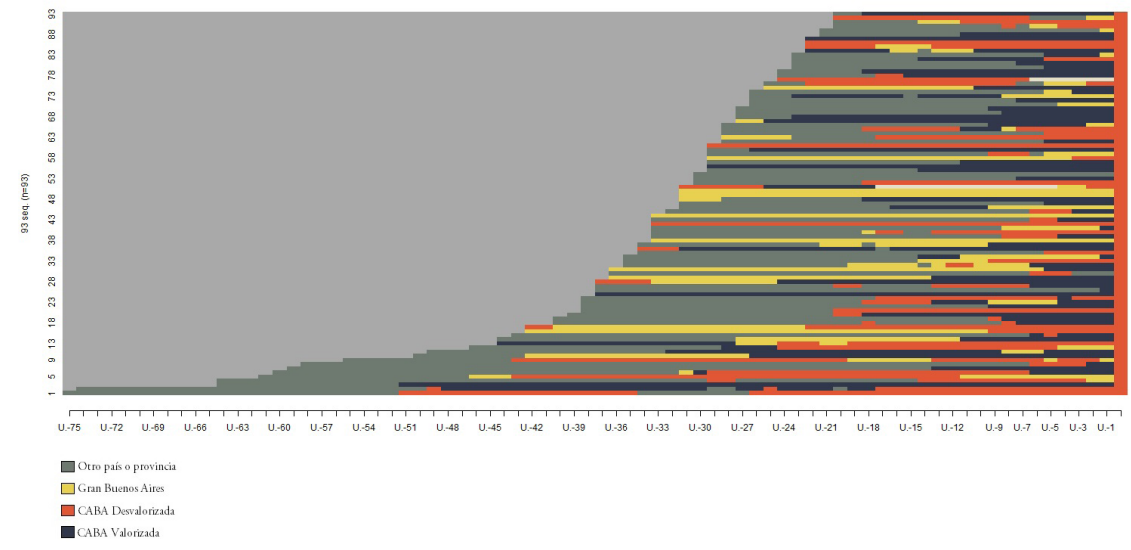

FUENTE: ELABORACIÓN PROPIA CON BASE EN LA ENCUESTA DE MOVILIDAD ESPACIAL (20I6-20I9)

Quienes llegan al Complejo Padre Mugica (y que, como vimos previamente, también habían demostrado, aunque en menor medida, su presencia residencial en las zonas más valiosas de la ciudad) muestran un mayor porcentaje de trayectorias ascendentes (Figura 8). Así, las trayectorias de los habitantes de Mugica presentan recorridos ascendentes de hogares que llegaron a las zonas más valorizadas desde 
otras áreas con menores externalidades urbanas, indicando conquistas en sus luchas por el acceso a la ciudad. Muchos de estos hogares vivieron durante gran parte de su vida en otros países o provincias y, con sus movimientos migratorios a la ciudad, provocaron una mejora en su posición residencial (Figura 7). Sus movimientos migratorios tuvieron como destino tanto los barrios más valorizados como los desvalorizados de la CABA, y en ambas situaciones implicaron mejoras en sus posiciones residenciales. Sin embargo, como veíamos previamente, estas mejoras de posición fueron acompañadas por una desmejora en los mecanismos de acceso al suelo y a la vivienda, ya que recurrieron principalmente a tipos de hábitats informales.

FIGURA 8 Tipos de trayectorias de localización residencial de los habitantes de Padre Mugica, en porcentajes

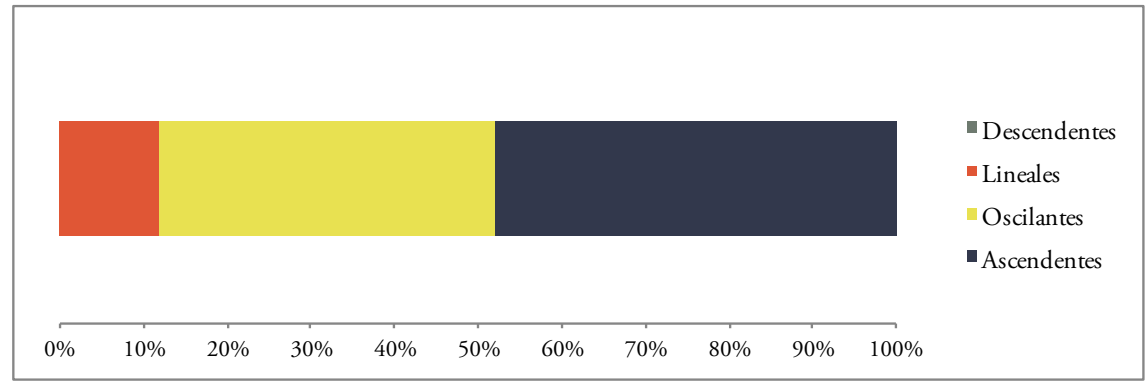

FUENTE: ELABORACIÓN PROPIA CON BASE EN LA ENCUESTA DE MOVILIDAD ESPACIAL (20I6-20I9)

Junto a las trayectorias ascendentes, al igual que en los casos de hogares que llegan a $\mathrm{AU} 3$, un gran porcentaje de tales recorridos también son oscilantes, dando cuenta de itinerarios inestables donde están presentes trayectos ascendentes y descendentes, visibilizando la inestabilidad y fragilidad de las posiciones urbanas que se lograron alcanzar.

\section{Llegar a la vivienda social: ‘̨avances o retrocesos en el acceso a la ciudad?}

Tal como fue mencionado al inicio, los dos barrios de vivienda social aquí analizados se ubican en dos zonas de la ciudad con características contrastantes, que habilitan condiciones diferenciales respecto al acceso a externalidades urbanas para sus habitantes. Ahora bien, el ingreso a la vivienda social y, en consecuencia, a estas localizaciones diferenciales, pudo implicar mejoras, desmejoras o incluso la reproducción de las posiciones que los destinatarios ocupaban previamente en la estructura urbana. En este sentido, la localizacion de los dos barrios de vivienda social no solo puede evaluarse en términos relativos, según la posición que ocupan en la estructura urbana, sino que también puede ser considerada en el marco de las trayectorias previas de sus habitantes. ¿En qué medida el acceso a la vivienda social implicó una mejora (o no) en las posiciones que estos hogares ocupaban previamente en la ciudad? ¿Qué papel desempeńa la llegada a la vivienda social en las luchas por el espacio urbano? 
FIGURA 9 | Efecto de la localización de la vivienda social sobre las posiciones urbanas anteriores, según barrio

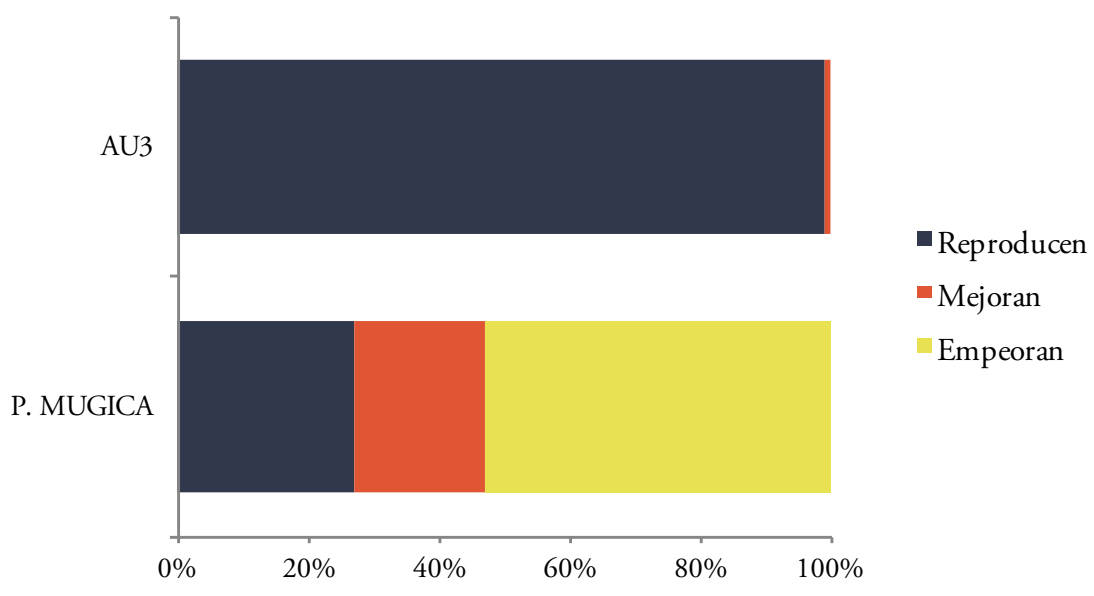

FUENTE: ELABORACiÓN PROPIA CON BASE EN LA ENCUESTA DE MOVILIDAD ESPACIAL (20I6-20I9)

Es interesante señalar que, con independencia de la localización de las viviendas, en ninguno de los casos el ingreso a la vivienda social significó principalmente una mejora respecto de las posiciones urbanas de sus habitantes (Figura 9). Estas políticas, a pesar de contar con la posibilidad y potencialidad de promover el acceso a la ciudad, modificando las posiciones urbanas de los hogares que se ven imposibilitados de acceder por vías del mercado a las localizaciones con mejores externalidades urbanas, terminan demostrando una tendencia fuertemente reproductora de la inscripción territorial de sus destinatarios. En el caso de AU3, la contundente mayoría de los casos reproduce las posiciones que ya habían alcanzado previamente en una zona disputada de la ciudad, por lo que la vivienda social, si bien presenta efectos reproductores, en cierta medida funciona como un canal de garantía de permanencia.

Sin embargo, la característica estrictamente deficitaria de la localización de la vivienda social ubicada en el eje sur conlleva necesariamente una lectura negativa del elevado porcentaje de hogares que, pese a ser destinatarios de la vivienda social, reproducen una posición desfavorable en la estructura urbana, que implica una desventaja en el acceso a externalidades urbanas positivas. Pero, además y a contramano de lo esperado, el acceso a la vivienda social ubicada en el sur de la ciudad terminó afectando en mayor medida de manera negativa a sus habitantes. El 53\% de quienes llegan a la vivienda social del barrio Padre Mugica empeoran su localización, por lo que esta intervención terminó imprimiendo un efecto negativo sobre las estructuras de oportunidades de estos hogares.

Este impacto negativo asume mayor relevancia al identificar que el efecto negativo que implicó llegar al barrio Padre Mugica fue relativamente mayor para quienes habían desarrollado trayectorias residenciales ascendentes (Figura 10). Se trata principalmente de situaciones de relocalización desde villas y asentamientos ubicados 
en barrios más centrales y de mayor valorización, áreas a las que habían accedido dibujando trayectorias residenciales ascendentes, que retroceden con la llegada a la vivienda social.

\section{FIGURA Io | Efecto de la vivienda social sobre las posiciones urbanas anteriores, según trayectoria residencial y barrio}

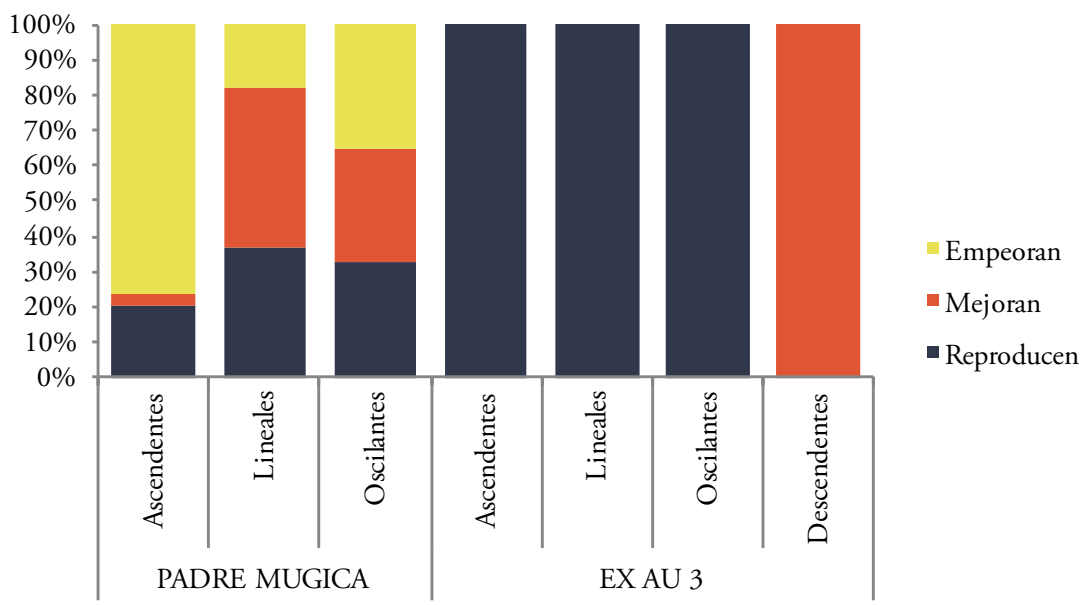

FUENTE: ELABORACIÓN PROPIA CON BASE EN LA ENCUESTA DE MOVILIDAD ESPACIAL (20I6-20I9)

$\mathrm{Si}$, como habíamos visto anteriormente, las trayectorias residenciales de quienes llegaron a la vivienda social Padre Mugica habían sido predominantemente ascendentes y habían logrado conquistar, a lo largo de las mismas, mejores posiciones urbanas, la localizacion de estas viviendas en el eje sur de la ciudad no posibilitó una nueva conquista. Por el contrario, produjo estancamientos e incluso retrocesos en estas trayectorias ascendentes. Esta última mudanza, impulsada por el Estado, imposibilitó continuar sus recorridos ascendentes e incluso resguardar las posiciones previamente alcanzadas, significando principalmente una desmejora locacional.

Por otro lado, como veíamos más arriba, el $20 \%$ de quienes llegaron a la vivienda social en el sur mejoraron su posición. Se trata principalmente de quienes habían desarrollado trayectorias de tipo oscilante y para quienes esta nueva residencia, antes que indicar la conquista de una mejor posición, demostró la recuperación de posiciones previamente alcanzadas y luego perdidas (Figura 10). En este sentido, al igual que en el caso de $\mathrm{AU}_{3}$, la vivienda social opera resguardando posiciones alcanzadas y posiblemente frenando en el futuro la inestabilidad que caracterizó a estos recorridos.

\section{Reflexiones finales}

Como mencionamos al inicio, la definición del lugar que ocupa la vivienda social conlleva la potencialidad de construir un orden urbano menos desigual y de facilitar mejores oportunidades urbanas para los sectores populares. Las políticas 
"tradicionales" han recibido fuertes críticas por sus efectos sobre el recrudecimiento de la desigualdad urbana, en gran medida debido a la localización deficitaria de las soluciones habitacionales promovidas. Como respuesta, en décadas recientes, las políticas en la región incorporaron la preocupación en torno a los "efectos del lugar" de la vivienda social, introduciendo nuevas localizaciones.

Los resultados de esta investigación situada en la Ciudad de Buenos Aires señalan que estas intervenciones, a pesar de incorporar "nuevos lugares para la vivienda social”, continúan impulsando patrones fuertemente reproductores de la estructura urbana desigual. Estas políticas, aparentemente novedosas, se han mostrado incapaces de transformar (de manera positiva) las posiciones que los sectores populares han ocupado históricamente en la ciudad. En el contexto local, además, las nuevas soluciones habitacionales producidas en "localizaciones positivas" han sido cuantitativamente menores que aquellas ubicadas en las zonas deficitarias y tradicionalmente priorizadas para la vivienda social, seńalando con ello un alcance muy limitado.

A lo largo de este trabajo se ha interrogado el papel que dos soluciones habitacionales recientes emplazadas en dos zonas diferenciadas de la Ciudad de Buenos Aires, desempeñaron sobre la inserción territorial de sus destinatarios. Se buscó conocer el efecto de la mudanza a estos dos barrios de vivienda social -es decir, a dos localizaciones contrastantes- sobre las trayectorias residenciales que sus habitantes habían desarrollado a lo largo de sus vidas. Llamativamente, la investigación demostró que la "nueva" vivienda social -independientemente de su localizaciónes incapaz de mejorar las oportunidades urbanas de sus destinatarios y generar efectos positivos sobre sus trayectorias residenciales. Asimismo, la característica excepcional de las nuevas localizaciones para la vivienda social, frente a la continuidad (mayoritaria) de la localización tradicional, sugiere la persistencia de una contradicción entre los lugares en que el Estado considera que los sectores populares deben vivir y aquellos que estos hogares han "escogido" para residir a lo largo de su vida a través de diversas estrategias.

Sin embargo, si bien en ninguno de los dos casos analizados la llegada a la vivienda social impactó positivamente sobre las trayectorias de sus habitantes, estas localizaciones contrastantes habilitaron efectos diversos sobre los derroteros de estas luchas por el acceso a la ciudad.

Las soluciones habitacionales ubicadas en localizaciones deficitarias han generado en gran medida retrocesos en las trayectorias residenciales de sus habitantes. Estos hogares, que en el marco de sus luchas biográficas por el acceso a la ciudad habían logrado inscribirse -aunque de manera informal- en zonas más favorecidas mediante trayectorias ascendentes, tras ser destinatarios de la acción estatal sufren un retroceso de sus conquistas urbanas. Incluso, la localización de esta solución habitacional evidenció la voluntad de ignorar los repertorios geográficos residenciales de sus destinatarios, asignándoles una posición residencial muy distante de aquellos lugares que habían sido priorizados por estos hogares como espacio de vida a lo largo de sus trayectorias.

Por otro lado, las soluciones ubicadas en zonas con acceso a externalidades urbanas generaron principalmente un efecto reproductor de las posiciones 
previamente alcanzadas. Es decir, que pese a ubicarse en lugares "novedosos" que podrían haber habilitado el goce de oportunidades urbanas mediante el acceso de los sectores populares hasta entonces excluidos de tales zonas, estas viviendas sociales se limitaron a "formalizar" la presencia de los sectores populares que ya residían allí. Incluso, el Estado tampoco ha demostrado una voluntad política de construcción de vivienda social en esta zona con el propósito de resguardar las posiciones urbanas de los hogares ocupantes, sino que estas viviendas han sido el resultado de un largo proceso de lucha de los mismos hogares frente a sucesivos intentos de desalojo por parte del Estado.

Los resultados de este trabajo problematizan la emergencia de un paradigma realmente disruptivo en las recientes políticas de vivienda social que logre modificar el lugar asignado históricamente a la vivienda social y a los sectores populares, así como transformar la estructura urbana hacia un orden menos excluyente.

\section{Referencias bibliográficas}

Ballent, A. (1998). La vivienda masiva. Salvación y caída de la arquitectura del siglo xx. 47 al fondo, 2(3), 2-7. http://sedici.unlp.edu.ar/handle/10915/44253

Ballent, A. (2018). Estado, política y vivienda entre dos peronismos: los grandes conjuntos habitacionales y las acciones en villas miseria en Buenos Aires, 1946-1976. Estudios Interdisciplinarios de América Latina y el Caribe, 29(1), 34-59. http://www3.tau.ac.il/ ojs/index.php/eial/article/view/1559

Baumont, C. (2007). Neighborhood effects, urban public policies and housing values: a spatial econometric perspective. Université de Bourgongne. https://citeseerx.ist.psu.edu/ viewdoc/download?doi=10.1.1.584.3401\&rep=rep1\&type=pdf

Bourdieu, P. (1999). Efectos de lugar. En P. Bourdieu (Dir.), La miseria del mundo (pp. 119124). Fondo de Cultura Económica.

Carman, M., Vieira, N. \& Segura, R. (2013). Antropología, diferencia y segregación urbana. En M. Carman, N. Vieira \& R. Segura (Coords.), Segregación y diferencia en la ciudad (pp. 11-34). FlacsoEcuador / Clacso / Ministerio de Desarrollo Urbano y Vivienda, Ecuador. http://biblioteca.clacso.edu.ar/clacso/gt/20140707123234/ segregacionydiferencia.pdf

Cavalieri, M., Gerscovich, A. \& Wainstein-Krasuk, O. (2010). Gestión social de vacíos urbanos en la Ciudad Autónoma de Buenos Aires. Un caso de estudio. Pampa: Revista Interuniversitaria de Estudios Territoriales, 1(6), 59-87. https://doi.org/10.14409/ pampa.v1i6.3177

Clark, W. A. V. (1982). Recent research on migration and mobility: a review and interpretation. Progress in Planning, 18, 1-56. https://doi.org/10.1016/0305-9006(82)90002-2

Consejo Económico y Social de la Ciudad de Buenos Aires (CESBA). (2013). Diagnóstico sociohabitacional de la ciudad de Buenos Aires. Consejo Económico y Social de la CABA. https://bit.ly/3fvm2YW 
Del Río, J. P. (2010). El lugar de la vivienda social en la ciudad: Una mirada desde el mercado de localizaciones intra-urbanas y las trayectorias habitacionales de los destinatarios. Presentación en III Jornadas del Doctorado en Geografia, 29 y 30 de septiembre de 2010, La Plata, Argentina. http://sedici.unlp.edu.ar/handle/10915/29909

Del Río, J., Vértiz, F. \& Ursino, S. (2014). La acción pública en el espacio urbano. Debates y reflexiones en torno a la noción de política urbana. Revista Estudios Sociales Contemporáneos, (11), 76-86. https://core.ac.uk/download/pdf/61890423.pdf

Di Virgilio, M. M. (2007). Trayectorias residenciales y estrategias habitacionales de familias de sectores populares y medios en Buenos Aires. Tesis de doctorado, Facultad de Ciencias Sociales, Universidad de Buenos Aires, Argentina.

Di Virgilio, M. M. \& Najman, M. (2019). Espacio de vida y tiempo de vida. El enfoque biográfico aplicado a la indagación de procesos urbanos. En E. Meccia (Dir.), Biografías y sociedad. Métodos y perspectivas (pp. 387-423). Ediciones unL (Universidad Nacional del Litoral) - Eudeba. https://bibliotecavirtual.unl.edu.ar:8443/bitstream/ handle/11185/5515/biografiasociedad.pdf?sequence=1 \&isAllowed $=y$

Di Virgilio, M. M., Najman, M. \& Brikman, D. (2019). Génesis de las desigualdades territoriales: una mirada histórica de los procesos de configuración de las antiguas periferias de la Ciudad de Buenos Aires. Andamios, 16(39), 47-76. https://doi. org/10.29092/uacm.v16i39.674

Dieleman, F. M. (2001). Modelling residential mobility; a review of recent trends in research. Journal of Housing and the Built Environment, 16(3-4), 249-265. https://doi. org/10.1023/A:1012515709292

Dunowicz, R. \& Boselli, T. (2009). Habitar en la vivienda social de Buenos Aires, 1905-2002. En J. M. Borthagaray (Comp.), Habitar Buenos Aires: Las manzanas, las casas y los lotes. Facultad de Arquitectura, Diseño y Urbanismo, Universidad de Buenos Aires (FADU-UBA).

Dureau, F. (2003). Sistemas residenciales: conceptos y aplicaciones. Territorios, (10-11), 41-70. https://doi.org/10.12804/revistas.urosario.edu.co/territorios/a.6010

Fachelli, S., Goicoechea, M. E. \& López-Roldán, P. (2015). Trazando el mapa social de Buenos Aires: dos décadas de cambios en la Ciudad. Población de Buenos Aires, 21(12), 7-39. https://ddd.uab.cat/pub/artpub/2015/132095/pobbueair_a2015n21p7iSPA_ postprint.pdf

Ferme, N., Raspall, T. \& Zapata, C. (2017). Los impactos de la política de construcción de vivienda social sobre la configuración territorial de la Ciudad de Buenos Aires, 1907-2014. En M. C. Rodríguez \& M. M. Di Virgilio (Comps.), Territorio, políticas habitacionales y transformaciones urbanas en las principales áreas metropolitanas de Argentina, 2004-2015 (pp. 75-116). Espacio Editorial.

Flores, C. A. (2008). Residential segregation and the geography of opportunities: a spatial analysis of heterogeneity and spillovers in education. University of Texas, Austin.

Gauthier, J. A., Widmer, E. D., Bucher, P. \& Notredame, C. (2010). Multichannel Sequence Analysis Applied to Social Science Data. Sociological Methodology, 40(1), 1-38. https:// doi.org/10.1111/j.1467-9531.2010.01227.x

Girola, M. F. (2008). Modernidad histórica, modernidad reciente. Procesos urbanos en el Área Metropolitana de Buenos Aires: los casos del Conjunto Soldati y Nordelta. Tesis de doctorado, Facultad de Filosofía y Letras, Universidad de Buenos Aires, Argentina. 
Girola, M. F. \& Thomasz, A. G. (2013). Del "derecho a la vivienda" al "derecho a la cultura": reflexiones sobre la constitución del "derecho a la ciudad" en Buenos Aires desde una perspectiva etnográfica. Anuário Antropológico, (II), 131-163. https://doi.org/10.4000/ aa. 593

Gorelik, A. \& Silvestri, G. (1991). Imágenes al sur. Sobre algunas hipótesis de James Scobie para el desarrollo de Buenos Aires. Seminario de Crítica, 17, Instituto Arte Americano e Investigaciones Estéticas. http://www.iaa.fadu.uba.ar/publicaciones/critica/0017.pdf

Harvey, D. (1977). Urbanismo y desigualdad social. Siglo xxI.

Harvey, D. (2012). Del derecho a la ciudad a la revolución urbana. Akal.

Hidalgo, R. (2007). ¿'Se acabó el suelo en la gran ciudad?: Las nuevas periferias metropolitanas de la vivienda social en Santiago de Chile. Revista EURE - Revista de Estudios Urbano Regionales, 33(98), 57-75. http://dx.doi.org/10.4067/S0250-71612007000100004

Hidalgo, R., Zunino, H. \& Álvarez, L. (2007). El emplazamiento periférico de la vivienda social en el área metropolitana de Santiago de Chile: consecuencias socio espaciales y sugerencias para modificar los criterios actuales de localización. Scripta Nova. Revista Electrónica de Geografía y Ciencias Sociales, 11(245), 27. http://www.ub.edu/geocrit/ sn/sn-24527.htm

Jirón, P. \& Imilan, W. (2018). Moviendo los estudios urbanos. La movilidad como objeto de estudio o como enfoque para comprender la ciudad contemporánea. Quid 16, (10), 17-36. http://repositorio.uchile.cl/handle/2250/153116

Kaztman, R. (1999). Marco conceptual sobre activos, vulnerabilidad y estructura de oportunidades. Documento preparado por la Oficina de Cepal en Montevideo, con el apoyo financiero del PNUD. https://repositorio.cepal.org/bitstream/handle/11362/28663/ lcmvd176rev1_es.pdf?sequence=1\&isAllowed=y

Kaztman, R. (2000). Notas sobre la medición de la vulnerabilidad social. En $5^{\circ}$ Taller Regional: La medición de la pobreza: métodos y aplicaciones (Continuación), 6 al 8 de junio de 2000, Aguascalientes, México. Comisión Económica para América Latina y el Caribe (CEPAL). https://ucu.edu.uy/sites/default/files/facultad/dcsp/vulnerabilidad_social.pdf

Marcos, M., Mera, G. S. \& Di Virgilio, M. M. (2015). Contextos urbanos de la Ciudad de Buenos Aires: una propuesta de clasificación de la ciudad según tipos de hábitat. Papeles de Población, 21(84), 161-196. http://www.scielo.org.mx/scielo.php?script=sci_ arttext\&pid=S1405-74252015000200007\&lng=es\&tlng=es

Massey, D. (2005). La filosofía y la política de la espacialidad: algunas consideraciones. En L. Arfuch, (Comp.), Pensar este tiempo: espacios, afectos, pertenencias (pp. 101-128). Paidós.

Massidda, A. (2011). Urban planning and undertakings upon informal settlements in Buenos Aires: A 1955-1959 Review. Master of Philosophy dissertation, University of Cambridge.

Najman, M. (2017). El nacimiento de un nuevo barrio: El caso del Conjunto Urbano Padre Mugica en la ciudad de Buenos Aires y sus impactos sobre las estructuras de oportunidades de sus habitantes. Territorios, (37), 123-155. https://doi.org/10.12804/ revistas.urosario.edu.co/territorios/a.4978

Najman, M. (2020). ¿Vivir mejor? Análisis de las trayectorias hacia la vivienda social y las transformaciones sobre las situaciones de exclusión de sus habitantes. Tesis de doctorado, Facultad de Ciencias Sociales, Universidad de Buenos Aires, Argentina. 
Oszlak, O. (1991). Merecer la ciudad: los pobres y el derecho al espacio urbano. Humanitas, Estudios CEDES.

Pérez Ripossio, R. (2013). El entramado conflictivo de la traza de la AU 3. Presentación en $x$ Jornadas de Sociologia, Facultad de Ciencias Sociales, Universidad de Buenos Aires, Argentina. https://cdsa.aacademica.org/000-038/202.pdf

Pooley, C. G. (1997). Introduction to housing strategies in Europe. En C. G. Pooley (Ed.), Housing strategies in Europe 1880-1930 (pp. 1-10). Leicester University Press.

Properati. (2019). Reporte del mercado inmobiliario - Properati + Oikos Bs.As. Agosto 2019. https://blog.properati.com.ar/reporte-del-mercado-inmobiliario-properati-oikos-bsas-agosto-2019

Ritschard, G. \& Studer, M. (Eds.). (2018). Sequence analysis and related approaches: Innovative methods and applications (Vol. 10). Springer.

Rodríguez, A. \& Sugranyes, A. (2005). El problema de vivienda de los "con techo". En A. Rodríguez \& A. Sugranyes (Coords.), Los con techo, un desafio para la politica de vivienda social (pp. 59-78). Ediciones Sur. http://www.sitiosur.cl/r.php?id=81

Rodríguez, A. \& Sugranyes, A. (2011). Vivienda privada de ciudad. Revista de Ingeniería, (35), 100-107. http://dx.doi.org/10.16924\%2Friua.v0i35.154

Rodríguez, M. C. (2005). Como en la estrategia del caracol: ocupaciones de edificios y politicas locales de hábitat en la ciudad de Buenos Aires (Vol. 2). El Cielo por Asalto.

Rodríguez, M. C. (2012). La ecuación vivienda-trabajo en las políticas argentinas del nuevo milenio. En J. Erazo (Coord.), Politicas de empleo y vivienda en Sudamérica (pp. 119144). FLACSO-Ecuador / CLACSO / Instituto de la Ciudad.

Rodríguez, M. C. \& Di Virgilio, M. M. (2011). Coordenadas para el análisis de las políticas urbanas: un enfoque territorial. En M. C. Rodríguez \& M. M. Di Virgilio (Coords.), Caleidoscopio de las politicas territoriales. Un rompecabezas para armar (pp. 17-46). Prometeo.

Rodulfo, M. B. (2008). Políticas habitacionales en Argentina: estrategias y desafíos. Programa Capacitación Técnicos y Profesionales del ivpba [Instituto de la Vivienda, Provincia de Buenos Aires].

Rodulfo, M. B. \& Boselli, T. (2015). Política habitacional en Argentina y desigualdades territoriales. Vivienda y Ciudad, (2), 30-41. https://revistas.unc.edu.ar/index.php/ ReViyCi/article/view/13173/13370

Ruiz-Tagle, J. \& Romano, S. (2019). Mezcla social e integración urbana: aproximaciones teóricas y discusión del caso chileno. Revista INVI, 34(95), 45-69. http://dx.doi. org/10.4067/S0718-83582019000100045

Slater, T. (2013). Your life chances affect where you live: A critique of the 'cottage industry' of neighbourhood effects research. International Journal of Urban and Regional Research, 37(2), 367-387. https://doi.org/10.1111/j.1468-2427.2013.01215.x

Snitcofsky, V. (2013). Impactos urbanos de la Gran Depresión: el caso de Villa Desocupación en la Ciudad de Buenos Aires (1932-1935). Cuaderno Urbano, 15, 93-109. https:// doi.org/10.30972/crn. 1515518

Varela, O. \& Fernández Wagner, R. (2012). El retorno del Estado. En C. Cravino (Comp.), Construyendo barrios. Transformaciones socioterritoriales a partir de los Programas Federales de Vivienda en el Área Metropolitana de Buenos Aires (2004-2006) (pp. 15-48). Ediciones Ciccus / Universidad Nacional de General Sarmiento, Argentina. 
Vergara Erices, L. (2019). Mixtura y cohesión social de barrio: una aproximación socio-espacial a las nuevas políticas de vivienda de Latinoamérica. Andamios, 16(40), 275-298. https://doi.org/10.29092/uacm.v16i40.707

Zapata, M. C. \& Belluscio, S. (2018). De la Autopista 3 al Barrio Parque Donado-Holmberg (Buenos Aires, Argentina): Una larga disputa por el espacio urbano. Quid 16, (9), 6090. https://publicaciones.sociales.uba.ar/index.php/quid16/article/view/2807

Ziccardi, A. \& Mier y Terán, A. (2015). Desigualdades urbanas y acción pública local en la Ciudad de México. En A. Sehtman \& E. Zenteno (Coords.), Continuidades, rupturas y emergencias. Las desigualdades urbanas en la América Latina (pp. 283-300). Universidad Nacional Autónoma de México (UnAM), Centro de Investigaciones sobre América Latina y el Caribe. http://ru.iis.sociales.unam.mx/jspui/handle/IIS/5657 